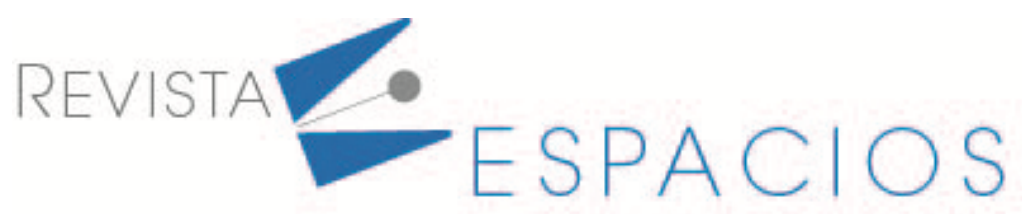

\title{
La innovación organizacional examinada desde las teorías del diseño, el cambio, la cognición y aprendizaje organizacionales
}

\section{Organizational innovation examined from the theories of organizational design, change, cognition and learning}

\author{
PERILLA, Ricardo B. ${ }^{1}$ \\ GONZÁLEZ, Mónica B. ${ }^{2}$
}

\begin{abstract}
Resumen
El presente artículo pretende revisar los fundamentos teóricos de la innovación organizacional a la luz de las corrientes teóricas desde las cuales ha sido estudiado este campo del conocimiento, específicamente desde las teorías del diseño, el cambio y la cognición y el aprendizaje organizacionales. Se concluye que se ha estudiado de manera amplia la relación entre la estructura organizacional y la innovación. Además, que el cambio organizacional, estructura, aprendizaje, creación de conocimiento e innovación, son elementos que se encuentran interrelacionados estrechamente.

Palabras clave: cambio \& innovación organizacional, teoría de la contingencia estructural, aprendizaje organizacional, creación de conocimiento.
\end{abstract}

\begin{abstract}
This article aims to review the theoretical foundations of "organizational innovation" in light of the theoretical currents from which this field of knowledge has been studied, specifically from the theories of design, change and cognition and organizational learning. It is found that the relationship between organizational structure and innovation has been studied extensively. In addition, it was concluded that organizational change, organizational structure, learning, knowledge creation and innovation, are elements that are closely interrelated.

key words: innovation, structural contingency theory, organizational change, organizational learning, knowledge creation
\end{abstract}

\section{Introducción}

El campo de la innovación organizacional ha venido cobrando relevancia en comparación al amplio estudio de la innovación tecnológica que a lo largo del tiempo se ha desarrollado. La innovación organizacional se ha estudiado desde diferentes perspectivas, para Wolfe (1994) existen tres corrientes a través de las cuales se ha desarrollado

\footnotetext{
${ }^{1}$ Profesor de tiempo completo del IDEAD en la Universidad del Tolima. Administrador de empresas y especialista en Gerencia de proyectos de la Universidad del Tolima, Magíster en Pensamiento estratégico y prospectiva. Estudiante del Doctorado en Administración de la Universidad Externado de Colombia. E mail: rbperillam@ut.edu.co

2 Profesora de tiempo completo del IDEAD en la Universidad del Tolima. Administradora industrial y especialista en Finanzas de la Universidad Pedagógica y Tecnológica de Colombia, Magíster en administración del Instituto Tecnológico de Estudios Superiores de Monterey. Estudiante del Doctorado en Gestión de la universidad EAN. E mail: mbgonzalezc@ut.edu.co
} 
la investigación sobre la misma: i) la difusión de la innovación, la cuál responde a la siguiente pregunta: ¿Cuál es el patrón de difusión de una innovación a través de una población de potenciales organizaciones adoptantes? ii) Innovación organizacional, responde a la pregunta ¿Qué determina la capacidad de innovación de la organización? iii) Teoría de procesos, que responde al cuestionamiento ¿Cuáles son los procesos por los que pasan las organizaciones en la implementación de innovaciones?

Por otro lado, Birkinshaw, Hamel, y Mol (2008) mencionan que existen cuatro perspectivas clave en la literatura sobre la innovación gerencial: i) la perspectiva institucional, la cual se enfoca en las condiciones socioeconómicas en las que se materializan las nuevas ideas y prácticas de gestión, ii) la perspectiva de la moda, la cual tiene como foco la interacción dinámica entre usuarios y proveedores de ideas de gestión, iii) la perspectiva cultural, que mira el nivel de reacción de la organización, ante la implementación de una nueva práctica de gestión, iv) la perspectiva racional, que se enfoca en cómo las innovaciones en la gestión producen mejoras en la eficacia de la organización.

Otras corrientes son las expuestas por Armbruster, Bikfalvi, Kinkel, y Lay, (2008); y Lam, (2004): i) las teorías del diseño organizacional que se ocupan de examinar la relación entre la estructura de la organización y la innovación, siendo la unidad de análisis la organización misma. ii) los estudios referentes al cambio y la adaptación organizacional, que buscan primordialmente comprender si las organizaciones pueden superar las inercias y lograr adaptarse a los cambios del ambiente. iii) las teorías de la cognición y el aprendizaje organizacional, la cual se centra en los fundamentos cognitivos de la innovación organizacional, los cuales se considera que están relacionados con la creación de conocimientos organizacionales.

En el presente artículo se pretende abordar las tres últimas corrientes: i) teorías del diseño organizacional ii) cambio y adaptación organizacional iii) cognición y aprendizaje organizacional. En la primera de ellas se tendrán en cuenta los postulados de la teoría de la contingencia estructural, considerando el rol que juega cada una de las contingencias: tamaño, estrategia y entorno. En la segunda, se tendrá en cuenta cómo ha emergido el cambio organizacional, sus etapas y evolución. Asimismo, se considerará como incide la implementación del cambio en la organización y los respectivos tipos de cambio, cambio dirigido, cambio planeado y cambio guiado, así como la relación del cambio organizacional con la innovación organizacional. En la tercera corriente teórica se cubrirá lo correspondiente al aprendizaje organizacional y la creación de conocimiento, y cómo interactúan estos dos factores con el fin de crear innovación en la organización, además del proceso de creación de conocimiento.

Finalmente, se examinará el significado de los conceptos organizational innovation, management innovation, administrative innovation y managerial innovation, teniendo en cuenta que para algunos autores, tienen el mismo significado, es decir, los ven como intercambiables (Damanpour y Aravind, 2011), sin embargo, para otros, existen algunas diferencias, sobre todo entre la amplitud y cobertura del concepto organizational innovation, frente a los demás (Camisón y Villar-lópez, 2012; Gu y Surendra, 2004).

\section{Metodología}

Para llevar a cabo la presente revisión, se procedió en primer lugar a seleccionar los artículos científicos tomados como base para examinar cada una de las teorías expuestas. Las bases de datos utilizadas fueron Jstor, Emerald Insight y Scopus. Luego de esto se revisó en profundidad a cada uno de los artículos seleccionados, para de esta manera poder realizar el correspondiente análisis y discusión. 


\section{Resultados y discusión}

\subsection{Teorías de la contingencia estructural}

La prospectiva Según Galbraith (2014), el diseño organizacional se ha desarrollado a partir de dos grandes corrientes de pensamiento; la primera de ellas con base en la publicación de Chandler (1962) "Strategy and Structure", quien sugiere que el tipo de estructura adoptado depende de la estrategia desarrollada en la organización; siendo este un diseño de arriba hacia abajo. La segunda corriente, denominada como el enfoque de los sistemas socio técnicos, desarrollada por Trist y Murray (1993), enfatiza en un desarrollo ascendente, tomando como base la tecnología necesaria para llevar a cabo las tareas y, de igual manera, el sistema social implicado para la ejecución de estas. Sin embargo, es necesario tener en cuenta que para Chandler (1962), es la estrategia, la contingencia que determina la estructura de la organización, excluyendo así otras contingencias que también tienen implicaciones importantes en la estructura organizacional adoptada. Pese a esto, con el paso del tiempo, las investigaciones sobre la estructura organizacional han pasado de considerar una sola contingencia, a tener en cuenta múltiples contingencias simultáneas (Donaldson, 2015).

En contraste con lo anterior, Pawlowski (2016) afirma que las dos corrientes sobre la interpretación de la estructura organizacional se clasifican en la clásica (tradicional) y la contemporánea. La primera de ellas "reduce la cuestión de la configuración de la estructura al organigrama y el alcance de las funciones y responsabilidades de los puestos y las dependencias orgánicas." (p. 2331). La segunda corriente tiene una mayor amplitud, puesto que abarca todo aquello que involucra el comportamiento de las personas dentro de la organización, por lo tanto, los cambios que se generen, darán lugar a diferentes dimensiones de la estructura organizacional.

Por otro lado, para Donaldson (2001), la teoría de la contingencia estructural plantea que la estructura de la organización debe adaptarse a situaciones particulares que se presentan bajo unas condiciones dadas, es decir, unas contingencias; estas son el entorno, el tamaño y la estrategia. Para Su, Chen, y Wang (2018), la contingencia del entorno incide sobre el tipo de estructura mecánica u orgánica, ya que una estructura mecánica se adapta mucho mejor a un entorno caracterizado por condiciones de estabilidad, puesto que la jerarquía por la que se distingue la estructura mecánica sería suficiente para unas condiciones de pocos cambios. Por el contrario, la estructura orgánica tendría un mejor desempeño bajo un entorno inestable, debido a que este tipo de estructura involucra una mayor participación para la innovación. A este mismo tipo de estructura (orgánica), Lawrence y Lorsch (1967) se refieren como de "bajo grado de estructura formalizada", mientras que a la mecánica, como "de un mayor grado de estructura" (p. 6).

En cuanto a la contingencia de tamaño, esta afecta el tipo de estructura ya sea burocrática o simple, por lo tanto, si una organización por su gran tamaño demanda una toma de decisiones rápida y acompañada de reglas definidas, lo más conveniente sería una estructura burocrática; por el contrario, una estructura simple (no burocrática), se adapta fácilmente a una organización pequeña que no está gobernada por reglas y es centralizada, es decir, la gerencia tiene la capacidad de tomar la mayor parte de las decisiones de manera oportuna (Donaldson, 2008).

La contingencia de la estrategia, por su parte, guarda estrecha relación con el tipo de estructura funcional y divisional. Una estructura funcional se adapta mejor a una organización no diversificada, es decir, organizaciones que se enfocan en un solo producto o servicio, y, por ende, es suficiente con las áreas funcionales de producción, marketing, recursos humanos, etc. Por su parte, la estructura divisional funcionaría mejor en una organización con una estrategia diversificada en cuanto a productos y mercados, por lo que se requiere de una rápida tasa de respuesta por parte de la gerencia ante las decisiones que habría que tomar en función de las diversas actividades que implican las diferentes líneas de productos (Donaldson, 2001). 
En este marco, la innovación se ha estudiado bajo la perspectiva de cuáles son aquellas variables estructurales de las organizaciones que la facilitan o la inhiben. En un estudio realizado en empresas manufactureras en la república de Korea, Tohirovich, Rhee, y Yoon (2017) encontraron que el comportamiento innovador de las personas actúa como mediador entre la centralización, la formalización y el desempeño de la innovación organizacional. Por otra parte, Marín-Idárraga y Cuartas (2016) en un estudio de Pymes, en Bogotá, concluyeron que el impacto de la estructura organizacional sobre la innovación es alto, cuando el análisis se realiza bajo las condiciones de la coalineación estratégica.

Un análisis más detallado lo realizaron Damanpour y Aravind (2012), quienes agrupan estos estudios empíricos en dos grandes olas, los anteriores a 1990 y los posteriores a 1990. En cuanto a los primeros, concluyen entre otras cosas que se apoya la perspectiva basada en Burns y Stalker (1961), de que las características relacionadas con la estructura orgánica facilitan en mayor grado la innovación, que la estructura mecanicista. Por otro lado, los resultados de la segunda ola soportan los hallazgos de un meta análisis realizado por Damanpour (1991), donde los resultados de la investigación empírica sobre las relaciones entre los factores organizacionales y la innovación, son principalmente consistentes.

Del análisis de Damanpour y Aravind (2012) se pueden concluir dos grandes aspectos, el primero, relacionado con la orientación del tipo de estructura dominante, pues en los estudios de la primera ola la distinción entre organizaciones innovadoras y no innovadoras se encontraba bajo la estructura orgánica y mecanicista, respectivamente. En contraste, durante la segunda ola se pasó de estructuras orgánicas y mecanicistas, hacia la estructura ambidiestra, esta entendida bajo diversos contextos, por ejemplo, exploración y explotación; proceso de adopción de la innovación, es decir, iniciación e implementación; el tipo de innovación, técnicas o administrativas; y la radicalidad de la innovación, radical o incremental. A pesar de esto, las diferencias entre estas estructuras duales, son análogas a las diferencias encontradas en las estructuras de las organizaciones innovadoras (orgánicas) y no innovadoras (mecanicistas) anteriores a 1990.

La segunda conclusión, relacionada con el efecto de la innovación en el desempeño de la organización, tiene una gran limitante, puesto que la gran mayoría de estudios han sido de corte transversal, por lo tanto, no es posible establecer la relación entre la innovación y el desempeño en la organización a lo largo del tiempo. Según Damanpour y Aravind (2012), tan solo dos investigaciones se enfocaron en examinar los efectos de la composición de los diferentes tipos de innovación en el desempeño de las empresas, estas coinciden en que "el desempeño organizacional sostenido es el resultado de la historia de la actividad de innovación de la empresa en lugar de un éxito de innovación ocasional." (p. 507).

Por otra parte, la estructura organizacional puede ser vista desde diferentes perspectivas, tales como la formalización, centralización y especialización (Olson, Slater, y Hult, 2005). Sin embargo, hay estudios que han abordado su análisis desde lo informal vs formal, es decir, haciendo la analogía, orgánica vs mecanicista; por ejemplo, Menguc y Auh (2010) en un estudio de firmas canadienses de alta tecnología, encontraron que las estructuras informales no ejercen un impacto en el desarrollo de las capacidades de innovación de productos, pero sí influyen en la ejecución de las capacidades de innovación de producto, en el rendimiento de nuevos productos.

Si bien, la teoría de la contingencia estructural ha sido la base para muchas investigaciones, esta ha recibido algunas críticas relacionadas con algunos problemas que expone Schoonhoven (1981), como la falta de claridad, pues argumenta que no es una teoría en el sentido estricto, sino más bien una meta teoría o una estrategia orientadora. Además, debido a esta falta de claridad de los teóricos de la contingencia, se desvanece la posibilidad de que realmente se esté prediciendo una interacción empírica. Aunado a esto, Ogbonna y Harris (2003) mencionan que tanto la perspectiva organizacional clásica, como la contingencial han venido siendo 
criticadas tanto por ignorar la naturaleza evolutiva de las organizaciones, como por tratar a las organizaciones como si estuvieran separadas de las personas que las dirigen.

Por otra parte, Mintzberg (1981) plantea cinco configuraciones a partir de las cuales se puede estructurar una organización, con base en unos elementos comunes tales como la especialización de tareas, formalización de procedimientos, entrenamiento formal y adoctrinamiento requerido para el trabajo, agrupación de las unidades y su respectivo tamaño, etc. En la tabla 1 es posible notar las características de cada uno de los tipos de estructura y su correspondiente proclividad para facilitar la innovación. De acuerdo con esto, el tipo de estructura "adhocrática" sería la ideal para la innovación, puesto que el poder cambia constantemente no en función de la autoridad, sino de la necesidad de toma de decisiones.

Tabla 1

Cinco configuraciones de Mintzberg

\begin{tabular}{|c|c|c|c|c|}
\hline Estructura simple & $\begin{array}{c}\text { Burocracia de la } \\
\text { máquina }\end{array}$ & $\begin{array}{l}\text { Burocracia } \\
\text { profesional }\end{array}$ & Forma divisional & Adhocrática \\
\hline $\begin{array}{l}\text { Se destaca por su } \\
\text { sencillez para operar } \\
\text { en entornos no } \\
\text { complejos, sin } \\
\text { embargo no es la } \\
\text { más adecuada para } \\
\text { innovaciones en } \\
\text { ambientes } \\
\text { turbulentos. }\end{array}$ & $\begin{array}{l}\text { Presenta como } \\
\text { principales } \\
\text { problemas el trabajo } \\
\text { repetitivo, } \\
\text { empleados } \\
\text { marginados y la } \\
\text { obsesión por el } \\
\text { control, aspectos que } \\
\text { obstaculizarían el } \\
\text { desarrollo de la } \\
\text { innovación en la } \\
\text { organización. }\end{array}$ & $\begin{array}{l}\text { Ideal para } \\
\text { organizaciones que } \\
\text { se encuentran en } \\
\text { entornos estables } \\
\text { pero complejos, por } \\
\text { lo tanto, no es el tipo } \\
\text { ideal de estructura } \\
\text { para innovar, sino } \\
\text { para mejorar lo } \\
\text { conocido. }\end{array}$ & $\begin{array}{l}\text { El sistema de control } \\
\text { adoptado desalienta } \\
\text { la asunción de } \\
\text { riesgos y la } \\
\text { respectiva } \\
\text { innovación, debido } \\
\text { principalmente a que } \\
\text { los directores de } \\
\text { cada división no } \\
\text { pueden actuar con } \\
\text { total libertad. }\end{array}$ & $\begin{array}{l}\text { Es compleja y no } \\
\text { estandarizada, es } \\
\text { muy fluida. funciona } \\
\text { muy bien en } \\
\text { entornos tanto } \\
\text { complejos como } \\
\text { dinámicos, por tal } \\
\text { razón es considerada } \\
\text { la estructura ideal } \\
\text { para el desarrollo de } \\
\text { la innovación. }\end{array}$ \\
\hline
\end{tabular}

Fuente: Elaboración propia con base en H. Mintzberg (1981)

Ahora bien, como se notó anteriormente, la estructura organizacional y su relación con la innovación ha sido ampliamente estudiada debido a que la estructura debe actuar como facilitadora de las actividades de innovación en la organización. Por lo tanto, el objetivo en este momento es comprender de qué manera la innovación organizacional se explica a través de la teoría de la contingencia estructural. Según Su et al. (2018), diferentes académicos han identificado los antecedentes de la innovación gerencial, clasificados en cuatro categorías: características organizacionales, factores gerenciales, factores estratégicos y factores del entorno. Precisamente dentro de las características organizacionales, se encuentra el estudio de la estructura organizacional. En el estudio de Su et al. (2018), se argumenta que la estructura organizacional de tipo orgánica, tiene un impacto positivo sobre la innovación gerencial debido a tres razones: la primera de ellas es que dado el carácter flexible de la estructura orgánica (descentralización en la toma de decisiones y falta de énfasis en las reglas y procedimientos), facilita la identificación de las debilidades en las capacidades y prácticas de gestión de la organización, mediante la participación tanto de gerentes como de empleados; permitiendo de esta manera el desarrollo de nuevas formas de mejorar y, por lo tanto, el inicio de la innovación en la gestión. La segunda es que la estructura orgánica por ser altamente flexible en la comunicación, facilita la absorción de nuevos conocimientos, la creación de conocimiento y el know how, de tal manera que redunde en la mejora de las capacidades y prácticas de gestión, promoviendo el desarrollo de la innovación en la gestión. Por último, debido a que la estructura orgánica pone poco énfasis en las reglas y procedimientos, esta fomenta la eliminación de la resistencia a la innovación, teniendo en cuenta que la innovación gerencial va acompañada de cambios en las reglas y procedimientos, lo cual generalmente provoca resistencia al cambio. 


\subsection{Teorías del cambio organizacional}

En el presente siglo, las organizaciones y los gerentes se enfrentan de manera más frecuente a las demandas de cambio que les impone el entorno. En todos los sectores de la economía, las presiones se encuentran relacionadas con la respuesta y anticipación ante las condiciones competitivas, tecnológicas, de mercado, económicas y sociales de continuo cambio (Buono y Kerber, 2010). Por lo tanto, una de las principales preocupaciones a nivel organizacional, es entender cómo responder de manera oportuna y efectiva, ante las diferentes condiciones cambiantes que son generadas externamente.

El cambio organizacional se ha venido estudiando desde diferentes perspectivas, Burgelman (1991) argumenta que alrededor del cambio organizacional se han desarrollado dos grandes debates a saber: el primero de ellos relacionado con el determinismo ambiental frente a la opción estratégica, el segundo, se sirve de la selección y la adaptación con el fin de explicar el cambio y la correspondiente supervivencia de las organizaciones. Por su parte, Hendrickson y Gray (2012 citando a Choi y Ruona 2011) afirman que las dos grandes perspectivas son las de la gestión estratégica y la del desarrollo organizacional.

Por otro lado, Romero, Matamoros, y Campo (2013 citando a Young 2009) enmarcan el cambio dentro de dos ámbitos: el cambio como tal y la forma como se expresa organizacionalmente; del primero dicen que las tipologías predominantes se dan en función a la velocidad, secuencia y continuidad. Del segundo, los temas comunes se relacionan con cambio y aprendizaje, cambios emergentes y desde las personas o desde los grupos.

En relación con estas perspectivas, es interesante el abordaje que le da Burnes (2005), ya que manifiesta que de los años 50's a los 80's el enfoque predominante fue el planeado, siendo Lewin (1947) su máximo exponente, sin embargo, este fue duramente criticado por la escuela Cultura-Excelencia, los posmodernistas y los procesualistas, tratando el enfoque de Lewin (1947) como lineal y estático. Por lo cual, la perspectiva CulturaExcelencia se enfocó en promover la flexibilidad en la cultura de la organización, promoviendo así la innovación y el emprendimiento mediante el cambio ascendente, continuo y cooperativo. Casi al mismo tiempo, según Burnes (2005), otros enfoques emergieron, tales como el de fijar la atención en el poder y la política para la toma de decisiones; el enfoque procesual y los modelos de equilibrio puntuado y el de transformación continua. Para dar paso, finalmente, a las teorías de la complejidad y su relación con el cambio organizacional.

Ahora bien, es necesario para el presente debate, considerar algunos conceptos acerca de lo que representa el cambio organizacional, lo que significa, sus implicaciones y alcance. Para Lines (2005), el cambio es “... deliberadamente planificado en la estructura formal, sistemas, procesos o dominio del mercado de productos de una organización con la intención de mejorar el logro de uno o más objetivos organizacionales" (pp. 9,10). Es claro que esta definición se encuentra dada desde una óptica de cambio planeado, intencional y que emerge desde arriba hacia abajo. En contraste, March (1981) ve el cambio organizacional como “...una ecología de respuestas concurrentes en varias partes de una organización a varias partes interconectadas del medio ambiente." (p. 564). Desde esta perspectiva, el cambio es visto como la respuesta de la organización ante las modificaciones presentadas por el entorno, y que, a su vez, depende de la magnitud con que el entorno realice tales modificaciones.

En relación con las clases de cambio, existen diferentes clasificaciones, que, si se analizan detenidamente, conducen a resultados concordantes. Por ejemplo, para Poole (2004), el cambio puede ser planificado o no planificado, gradual o radical, y recurrente o sin precedentes. Asimismo, puede ser planeado o emergente (Burnes, 2005), o en cuanto a niveles, superficial y en profundidad, o cambios de primer orden o transaccionales, y cambios de segundo orden o transformacionales (Sandoval Duque, 2014).

Uno de los grandes cuestionamientos que surge en relación al cambio organizacional, es ¿por qué ocurre este? En sintonía con los conceptos vistos anteriormente, se podría pensar que es debido a grandes cambios generados 
en el ambiente, transformaciones profundas que desencadenan la reacción debida por parte de la organización, como respuesta ante estos desequilibrios. Sin embargo, March (1981) afirma que la mayor parte "de los cambios en las organizaciones no son el resultado de procesos o fuerzas organizacionales extraordinarias, ni de una imaginación, persistencia o habilidad poco común, sino de procesos rutinarios y relativamente estables que relacionan a las organizaciones con su entorno" (564). Esto lo afirma en el contexto de las organizaciones burocráticas, las cuales se consideran ineficaces, pero, por lo general, son este tipo de organizaciones que se caracterizan por su competencia ordinaria y baja iniciativa las que se tienen en cuenta como objeto de estudio.

Por otro lado, Sandoval Duque (2014) expone dos grandes causas para que se de el cambio organizacional: causas externas y causas internas. Dentro de las primeras, se encuentran las nuevas tendencias de mercados, consumidores con diferentes necesidades, innovaciones tecnológicas, diferentes perfiles de los competidores y, en general, nuevos estilos de vida. En relación a las causas internas, estas tienen que ver con el desarrollo mismo de la organización, su visión de futuro, las modificaciones generadas en la estructura y procesos, entre otros.

Por otra parte, en relación con la implementación del cambio organizacional, Kerber y Buono (2005) argumentan que las bajas tasas de éxito que se obtienen en los procesos de cambio, es debido principalmente a un "desajuste entre los requisitos de la situación y el enfoque del cambio que se aplica." (p. 25). Por lo tanto, y teniendo en cuenta los tres enfoques de cambio ampliamente conocidos en la literatura: cambio dirigido, cambio planeado y cambio guiado; proponen que para que haya un proceso de implementación exitoso, la decisión sobre el tipo o enfoque de cambio a implementar, debe tomarse teniendo en cuenta dos contingencias situacionales a saber: la complejidad del negocio y la incertidumbre socio-técnica.

En consecuencia, en un escenario de baja complejidad y baja incertidumbre socio-técnica, el cambio dirigido sería suficiente para asegurar una implementación exitosa del mismo. Sin embargo, un impulsor para moverse del cambio dirigido hacia el cambio planeado, es que aumente la complejidad del negocio, considerando que el cambio planeado es más participativo, involucra en mayor grado a las personas y su liderazgo puede surgir desde cualquier nivel de la organización, en tanto que el cambio dirigido se basa en la autoridad y el cumplimiento, debido a que es impulsado desde el nivel más alto de la organización. Por otro lado, un impulsor que propicie el traslado del cambio dirigido hacia el cambio guiado, viene a ser la alta incertidumbre socio-técnica (Kerber y Buono, 2005).

Vale la pena destacar que el cambio guiado, tal y como lo describen Buono y Kerber, (2010) y Kerber y Buono, (2005), se genera a través de un proceso iterativo de interpretar y diseñar, implementar e improvisar, responsabilizarse y aprender del esfuerzo de cambio y, finalmente, compartir el aprendizaje a nivel de todo el sistema. Esto da como resultado una espiral de aprendizaje, innovación y desarrollo, lo cual permite obtener una mejora continua en los cambios existentes, así como en la capacidad de fomentar nuevos cambios y soluciones.

Pese a lo anterior, el mayor desafío para quienes lideran el cambio es el manejo de la resistencia, puesto que este pasa a ser uno de los elementos cruciales dentro del proceso de cambio en la organización (Bovey y Hede, 2001). Ahora bien, al analizar la resistencia al cambio, es necesario considerar dos clases de variables, contextuales e individuales. Las primeras relacionadas con el proceso de implantación en sí, el flujo de información o la participación permitida. Por otro lado, las individuales, tienen que ver con las características o personalidad del empleado involucrado en el cambio (García-Cabrera, Álamo-Vera, y Hernández, 2011). Sin embargo, es interesante analizar el abordaje que le dan a la resistencia al cambio Kerber y Buono, (2005), ya que para ellos, la resistencia “... puede considerarse, en parte, como una función del desajuste entre las demandas de la situación y el enfoque seleccionado para el cambio." (p. 34). Es decir, la discrepancia que se podría generar entre la elección del tipo de cambio (dirigido, planeado o guiado) y las contingencias situacionales presentes en la organización como la complejidad del negocio y la incertidumbre socio-técnica. 
Con respecto a la relación entre la innovación y el cambio, es claro que son dos campos de estudio estrechamente relacionados, los cuales generalmente son estudiados de manera conjunta, de tal manera que algunas veces, incluso, se tiene dificultad para visibilizar los límites entre uno y otro. En efecto, "ciertamente toda innovación en términos organizacionales es cambio. Aunque no todo cambio es innovación" (West y Farr, 1989 citado en Orengo, Grau, y Peiró, 2002, p. 9). En consecuencia, la innovación hace parte del cambio organizacional (Glynn, 1996; Orengo et al., 2002).

\section{El cambio organizacional y las teorías de selección}

El postulado principal que gira en torno a esta teoría se relaciona con el rol limitado o nulo que desempeñan las organizaciones en relación con el cambio, pues este ocurre más bien de acuerdo con la selección de las organizaciones que realiza el ambiente (Garcilazo, 2011). Esto sucede debido a que existen presiones inerciales que impiden el cambio por parte de la organización individual, dichas presiones inerciales pueden ser tanto internas como externas, dentro de las internas se encuentran la inversión en planta, equipos y personal especializado, los cuales no son fácilmente transferibles a otras actividades; las limitantes en cuanto a la información que reciben los tomadores de decisiones de la organización, esto hace que obtengan muchas veces, información incompleta; las limitaciones políticas internas, las cuales en caso de una redistribución de recursos por la necesidad de alterar la estructura de la organización, impiden el cambio o la adaptación. Finalmente, la propia historia de la organización puede generar inercia, puesto que las tareas y otras actividades, con el tiempo se convierten en acuerdos normativos, estos fortalecen la resistencia al cambio a manera de justificación y desechan alternativas de actuación (Hannan y Freeman, 1977).

En cuanto a las presiones externas se encuentran las barreras legales y fiscales a la entrada y salida de los mercados, limitando las posibilidades de adaptación; limitaciones para el acceso a la información externa, lo cual repercute en limitaciones de índole interna; limitaciones debido a la legitimidad externa, de modo que es difícil cambiar o adaptarse a nuevas situaciones cuando se puede ver en riesgo la pérdida de legitimidad; por último se encuentra el problema de la racionalidad colectiva, es decir, no existe la certeza de que una estrategia que sea óptima para una organización individual, lo sea para todas en general (Hannan y Freeman, 1977).

De todo lo anterior se podría inferir que dadas estas circunstancias, es casi nula la posibilidad que tienen las organizaciones para afrontar cambios de acuerdo con las exigencias del entorno, de hecho, las capacidades para innovar se verían altamente restringidas, pues lo que plantea esta teoría es que las organizaciones no cuentan con la capacidad para modificar en algún grado las condiciones que el entorno le suministra, de igual manera, se podría afirmar que las organizaciones entran en un estado en el cual tampoco logran llevar a cabo acciones para impedir caer en tal inercia a nivel interno, sobre la cual muy seguramente tendrían más posibilidades de gobernabilidad.

No obstante, Pfeffer (1997) argumenta que el concepto de inercia, en este contexto, no debe entenderse de manera absoluta, puesto que no se puede interpretar como la falta de cambio, sino más bien como la incapacidad de las organizaciones de cambiar a la misma velocidad de los cambios que se presentan en el ambiente.

\subsection{Teorías de la cognición y el aprendizaje organizacional}

El conocimiento para Drucker (2005), en esta sociedad, viene a ser el recurso primordial tanto para individuos como para la economía en general, donde los factores de producción tradicionales como la tierra, el capital y el trabajo, aun cuando no han desaparecido, toman un papel secundario, teniendo en cuenta que pueden ser adquiridos con facilidad.

Por su parte, Senge (1990) argumenta que aquellas organizaciones que logren aprovechar el compromiso y la capacidad de las personas para aprender en los diferentes niveles de la organización, serán aquellas que podrán 
sobre salir en el largo plazo; de hecho, las organizaciones de aprendizaje son posibles gracias a que las personas llevan en su naturaleza la inclinación por aprender.

Recientemente ha crecido el número de estudios que vinculan al conocimiento y el aprendizaje organizacional, con la innovación; Rezaei, Allameh, y Ansari (2018) encontraron una relación positiva entre el aprendizaje organizacional y las innovaciones tecnológicas y administrativas, así como el papel mediador del aprendizaje organizacional entre la creación de conocimiento y las innovaciones tecnológicas y administrativas. Liao y Wu (2010) hallaron que el aprendizaje organizacional actúa como variable mediadora entre la gestión del conocimiento y la innovación organizacional; además concluyen en su estudio que el aprendizaje organizacional afecta a la innovación organizacional. Por su parte, Sutanto (2017) halló que hay una influencia significativa de la capacidad de aprendizaje organizacional y la creatividad, sobre la innovación organizacional, en una muestra de instituciones de educación superior.

Ahora bien, el punto central radica en responder cómo a través del aprendizaje organizacional y la creación de conocimiento, se genera la innovación. Para Calantone, Cavusgil, y Zhao (2002), una organización que está comprometida con el aprendizaje puede mejorar su capacidad de innovación a través de tres maneras: si esta posee tecnología de punta y la utiliza para innovar; si tiene la habilidad para conocer y entender las necesidades de los clientes, y esta se refleja en los productos que saca al mercado; y finalmente, si monitorea las acciones de los competidores, tiene en cuenta sus fortalezas y debilidades y aprende de ellas. Sin embargo, no queda lo suficientemente claro, cómo es el proceso que debería llevarse a cabo para que se produzca la innovación.

Por otra parte, acerca del aprendizaje organizacional y cómo ocurre este, Argyris y Schön (1978) mencionan que es cuando los miembros de la organización actúan como agentes de aprendizaje, respondiendo a los cambios tanto internos como externos de la organización, que les permite a su vez detectar errores de la teoría en uso, incorporando los resultados de su investigación tanto en imágenes privadas, como en mapas compartidos de la organización. Es decir, en el momento en el que los individuos identifican fallas en las prácticas rutinarias de la organización, las correcciones de estas, por un lado, hacen que el individuo cree su imagen particular acerca de la misma, sin embargo, esto no es suficiente para que se traduzca en aprendizaje organizacional, es necesario que haya un método mediante el cual la organización logre a nivel general, tener la interpretación de este hecho, a esto se le conoce como el mapa compartido de la organización.

Pese a lo anteriormente expuesto, sigue quedando inconclusa la forma en la que la innovación organizacional se explica a través de la creación de conocimiento y el aprendizaje organizacional. Tal vez una corriente teórica que permita dar luces para la comprensión de este fenómeno es la expuesta por Nonaka (1994) en su teoría dinámica de la creación de conocimiento organizacional; en esta, la piedra angular radica en el conocimiento tácito. Para Grant (1996), el conocimiento tácito se puede distinguir del explícito en cuanto a que este último se relaciona más con hechos y teorías, en tanto que el tácito se identifica con el saber hacer; de igual manera, una distinción más clara se puede establecer mediante la transferibilidad del conocimiento, pues el explícito es posible transferirse mediante la comunicación, mientras que el tácito, como no puede ser codificado, solamente puede observarse a través de su aplicación.

La teoría de la creación de conocimiento de Nonaka (1994) se fundamenta en dos dimensiones: la epistemológica y la ontológica; esta última considera que finalmente, quienes crean conocimiento son los individuos, por lo tanto, una organización no puede crear conocimiento sin ellos. La dimensión epistemológica abarca los dos tipos de conocimiento, tácito y explícito, considerando que cada uno cuenta con sus particularidades y forma mediante la cual puede ser observado.

Ahora bien, para que haya creación de conocimiento, debe haber una interacción entre el conocimiento tácito y explícito de los individuos, lo que Nonaka (1994) denomina como conversión de conocimiento. Como resultado 
de esto, surgen cuatro formas de conversión del conocimiento: la primera, de conocimiento tácito a tácito, se le conoce como socialización; esta se lleva a cabo generalmente producto de compartir experiencias entre individuos, por ejemplo, entre maestros y aprendices, o incluso, entre el personal encargado de desarrollar productos y los clientes. La segunda, de conocimiento tácito a explícito, conocida como exteriorización, surge del diálogo o la reflexión colectiva y comúnmente desemboca en la creación de conceptos, mediante la combinación de la deducción y la inducción. La tercera, de conocimiento explícito a explícito, denominada como combinación, se puede afirmar que es la más visible y común de todas, puesto que se da como resultado del intercambio de información entre individuos; información que es explícita, representada en comunicaciones por distintos medios. Finalmente, la cuarta forma de conversión, de conocimiento explícito a tácito, conocida como interiorización, se presenta mediante el "aprendiendo haciendo", además, se trata de la interiorización del conocimiento adquirido a través de la socialización, exteriorización y la combinación.

Por lo tanto, "para que se de la creación de conocimiento organizacional es necesario que el conocimiento tácito acumulado en el plano individual se socialice con otros miembros de la organización, empezando así una nueva espiral de creación de conocimiento" (Nonaka y Takeuchi, 1995, p. 78). Sin embargo, el proceso no termina aquí, puesto que la espiral de conocimiento debe abarcar también la dimensión ontológica, tomando en cuenta los diferentes niveles, comenzando por el individual y avanzando hacia el grupal, organizacional, e incluso, el interorganizacional.

Teniendo en cuenta lo anterior, se debe advertir que la naturaleza dinámica de esta teoría radica en "...la interacción de estas dos espirales a través del tiempo. Este proceso dinámico es el que genera innovación" (Nonaka y Takeuchi, 1995, p. 262). Además, Nonaka y Takeuchi (1995) agregan que el conocimiento por sí solo no genera innovación, realmente es el proceso mediante el cual se crea el conocimiento, el que permite llevar a cabo actividades innovadoras dentro de la organización.

En continuidad con lo anterior, es necesario aclarar que algunos autores subrayan la importancia de no confundir conceptos como creación de conocimiento, gestión del conocimiento y transferencia de conocimiento, pues cada uno de ellos involucra aspectos particulares y diferenciadores. Li y Gao (2003) argumentan que "la transferencia de conocimientos no es creación de conocimientos. La transferencia de conocimientos se refiere a la emulación y el aprendizaje continuo de los competidores en el mercado o licenciados por los inventores." (p. 11). Con respecto a la gestión del conocimiento, Li y Gao (2003) argumentan que según Nonaka, el conocimiento no puede ser gestionado, sino dirigido. Además, insisten en que la tarea del gestor de conocimiento tiene que ver más con propiciar un ambiente adecuado para que los trabajadores del conocimiento lleven a cabo sus funciones bajo las mejores condiciones, por lo tanto, a su vez, una de las principales funciones del gestor de conocimiento es asegurarse de que los procesos de socialización, externalización, combinación e internalización (los cuatro modos de conversión de conocimiento de Nonaka, 1994) entre los individuos, se desarrollen adecuadamente.

Ahora bien, ante todo lo anteriormente expuesto sobre esta teoría hasta el momento, es válido cuestionarse: ¿Cuál es la relación entre el aprendizaje organizacional y la creación de conocimiento? Para Rezaei et al. (2018), la creación de conocimiento tiene un efecto positivo sobre el aprendizaje organizacional, por lo tanto "Este resultado sugiere que las empresas basadas en el conocimiento necesitan fomentar la creación de nuevos conocimientos si tienden a convertirse en organizaciones de aprendizaje." (p. 130). En tanto que para Nonaka (1994), el aprendizaje organizacional viene a ser solo una de los cuatro tipos de conversión del conocimiento, el de interiorización, en el cual como se mencionaba anteriormente, se produce la conversión de conocimiento explícito a tácito; por lo tanto, Nonaka (1994) ve la creación de conocimiento dentro un rango mucho más amplio que trasciende el alcance del aprendizaje, de hecho, acerca de este menciona: "Tomado por sí mismo, el aprendizaje tiene connotaciones más bien limitadas y estáticas, mientras que la creación de conocimiento 
organizacional es un concepto más amplio y dinámico." (p. 34). Es precisamente a partir de este dinamismo que es posible generar innovación.

Por otra parte, Nonaka y Takeuchi (1995) destacan las limitantes más sobresalientes en relación a la teoría del aprendizaje organizacional y su rol frente a la creación de conocimiento propuesta por ellos: la primera de ellas enfatiza en la ausencia que tiene la creación de conocimiento dentro de las teorías de aprendizaje organizacional, más aun cuando precisamente la creación de conocimiento es la base de estas teorías. La segunda limitante corresponde a la visión aún cerrada de percibir el aprendizaje organizacional como una metáfora del aprendizaje individual, lo cual genera un sesgo que termina por adoptar una perspectiva incorrecta del aprendizaje colectivo. En la tercera se hace alusión a que hay un consenso en que el aprendizaje organizacional corresponde a un proceso de cambio adaptativo, arraigado en rutinas y la memoria de la organización; lo cual impide una idea de creación de conocimiento dentro de estas teorías. Finalmente, la cuarta limitante se relaciona con el aprendizaje de doble ciclo, el cual resulta ser de difícil implantación para el aprendizaje organizacional, por lo cual, acuden al desarrollo organizacional como un soporte adicional, sin embargo, realmente este es insuficiente, puesto que nadie puede conocer de manera objetiva el método adecuado para el uso correcto del aprendizaje de doble ciclo; en contraste, la creación de conocimiento da por sentado que el aprendizaje de doble ciclo hace parte de la organización creadora de conocimiento.

Continuando con la pregunta planteada anteriormente, Merx-Chermin y Nijhof (2005) argumentan que la creación de conocimiento, el aprendizaje organizacional y la innovación, forman lo que denominan "modelo de innovación en espiral", este se explica de la siguiente manera: "Cuando la innovación se considera como la transformación de conocimientos valiosos en valor agregado para las partes interesadas, la generación de conocimientos es una condición necesaria para el proceso de innovación." (p.139); ahora bien, para que esto se lleve a cabo de manera eficiente, es necesario aprender a aprender. Dado que la información resultante de los dos procesos anteriores se convierte en nuevos conocimientos; el proceso de aprendizaje de la organización pasa a ser un requisito para la generación de conocimiento, lo cual desencadena un proceso cíclico y continuo.

Como se ha visto en la exposición de la presente teoría, campos de conocimiento como el aprendizaje organizacional y la creación de conocimiento organizacional, brindan valiosos fundamentos para comprender cómo se genera la innovación a nivel organizacional. Además de estos, también se encuentran otras variables que hacen parte de la cognición, y que pueden explicar en parte, la innovación organizacional, como por ejemplo, la creatividad a nivel individual, grupal y organizacional (Woodman, Sawyer, y Griffin, 1993), y la inteligencia (Glynn, 1996); sin embargo, como se ha visto, el enfoque ha recaído sobre el aprendizaje organizacional y la creación de conocimiento organizacional.

\subsection{Discusión}

La innovación organizacional aún cuando en la última década ha tenido un incremento importante en la atención académica (Simao y Franco, 2018), no se logra todavía la unificación, unicidad y homogenización de su concepto (Camisón-Zornoza, Lapiedra-Alcamí, Segarra-Ciprés, y Boronat-Navarro, 2004; Camisón y Villar-lópez, 2012; Lam, 2004; Su et al., 2018), ya que términos como managerial innovation, organizational innovation, administrative innovation y management innovation se superponen notablemente (Damanpour y Aravind, 2011), de hecho, uno de los principales obstáculos para la investigación en la innovación tiene que ver con la ambigüedad entre los autores acerca de los términos adoptados (Wolfe, 1994).

Teniendo en cuenta lo anterior, se hace necesario analizar los términos antes mencionados, con el fin de determinar si se refieren a lo mismo, o, por el contrario, existen diferencias en cuanto a alcance, profundidad o significado. La innovación organizacional ("organizational innovation") para Damanpour y Aravind (2011)

“...Incluye cambios en la estructura y los procedimientos internos de la organización que facilitan el cambio y el 
crecimiento de la organización. Estos cambios podrían deberse a la introducción de innovaciones radicales e incrementales" (p. 428). Al parecer esta definición es bastante general, y podría estar en concordancia con lo expuesto por Orengo et al. (2002), para quien la innovación tecnológica hace parte de la innovación organizacional.

Por otro lado, management innovation se refiere a "la invención e implementación de una práctica, proceso, estructura o técnica de gestión que sea nueva en el estado del arte y que tenga por objeto promover los objetivos de la organización." (Birkinshaw, Hamel, y Mol, 2008, p. 825). Hasta el momento, al parecer, no hay diferencias sustanciales entre los dos términos expuestos ("organizational innovation" vs "management innovation"), sin embargo, Camisón y Villar-lópez (2012) analizan la definición de innovación organizacional dada por la OECD (2005): "La innovación organizacional (IO) es la introducción de nuevos métodos organizativos para la gestión empresarial en el lugar de trabajo y/o en la relación entre la empresa y los agentes externos." (p. 1). Con respecto a esta, afirman que la misma considera dos dimensiones: inter e intra organizacional, es decir, cubre tanto lo que ocurre dentro de la organización, como lo que traspasa sus límites, como por ejemplo los acuerdos de cooperación. Por esta razón, Camisón y Villar-lópez (2012) concluyen que el concepto de innovación organizacional, no puede ser intercambiable con términos como management innovation, managerial innovation y administrative innovation; puesto que tiene un significado más amplio.

Ahora bien, ante la definición de management innovation expuesta anteriormente, Birkinshaw et al. (2008), plantean el cuestionamiento de qué tan nueva debe ser una innovación, teniendo en cuenta que la misma menciona que la invención debe ser nueva en el estado del arte, es decir, sin precedentes, sin embargo, para algunos autores esta puede ser nueva para la organización, aún cuando ya tenga cierta madurez en el mercado.

Con respecto al concepto managerial innovation, para Damanpour y Aravind (2011), este abarca los otros tres (organizational, administrative y management), y los trata como términos intercambiables, con la principal característica de que estos representan un tipo de innovación distinta a las innovaciones de producto/servicio y procesos tecnológicos; a su vez, Damanpour y Aravind (2011) presentan la siguiente definición de "managerial innovation": "...nuevos enfoques en el conocimiento para realizar el trabajo de gestión y nuevos procesos que producen cambios en la estrategia, estructura, procedimientos administrativos y sistemas de la organización" (pp. 431-432). Argumentando que esta definición representa los tres términos anteriores.

Con respecto al término "administrative innovation", Evan (1966) aclara que su distinción frente a la innovación técnica, se encuentra dentro de un marco más amplio, la estructura social y tecnológica; y la define como "...la implementación de una idea para una nueva política relativa a la contratación de personal, la asignación de recursos, la estructuración de tareas, de autoridad, de recompensas." (p. 51). Aclarando que las innovaciones administrativas hacen parte del sistema social de la organización. Por su parte, Birkinshaw et al. (2008) mencionan: "La innovación administrativa se refiere típicamente a una estrecha gama de innovaciones en torno a la estructura organizacional y las políticas de recursos humanos y no incluye las innovaciones en, por ejemplo, la gestión de la comercialización o de las operaciones" (p. 829). Esto lo argumenta, contrastándola frente a la innovación organizacional, de la cual dice que se utiliza para referirse a cualquier tipo de innovación generada por las organizaciones, inclusive, las innovaciones de productos. En este mismo sentido, Gu y Surendra (2004) comentan que la innovación organizacional se encuentra dividida en tres tipos: prácticas de producción y eficiencia, prácticas de gestión del recurso humano y prácticas relacionadas con la calidad del producto/servicio.

En consecuencia, y en la misma línea del argumento de Camisón y Villar-lópez (2012), al parecer, la innovación organizacional representa un concepto más amplio y que abarca más elementos de la organización, que la innovación administrativa y la innovación gerencial. 
A lo largo del presente artículo se expusieron tres teorías: contingencia estructural, cambio organizacional y cognición y aprendizaje organizacional; con el propósito de comprender, cómo, a través de cada una de ellas, se explica la innovación organizacional. Una vez analizadas estas tres grandes corrientes teóricas, se encuentra que las mismas se interrelacionan por medio de la innovación organizacional, en otras palabras, es la innovación organizacional el factor en el cual confluyen. Por ejemplo, Buono y Kerber (2010) dentro de los elementos fundamentales para el fortalecimiento de la capacidad de cambio organizacional, consideran como algo necesario, entre otros aspectos, la flexibilidad de la estructura organizacional para la creación de nuevos grupos; la creación de sistemas para compartir conocimientos, información y aprendizaje; y la dedicación de recursos para el escaneo continuo del entorno con el fin de obtener nuevas ideas, entre otros. Elementos que hacen parte de las otras dos corrientes teóricas. Además, cabe destacar que dentro del proceso de cambio guiado, Buono y Kerber (2010) enfatizan en que "La espiral resultante de aprendizaje, innovación y desarrollo contribuye tanto a la mejora continua de los esfuerzos de cambio existentes como a la capacidad de generar nuevos cambios y soluciones." (p. 8). Dejando entre ver que aprendizaje, conocimiento, estructura, cambio e innovación, son elementos que se encuentran interrelacionados y que deben operar conjuntamente en la organización.

En relación con la anterior espiral del cambio guiado, es interesante notar cómo esta guarda una estrecha similitud con las espirales de creación conocimiento expuesta por Nonaka y Takeuchi, (1995), desde lo ontológico y lo epistemológico, donde la innovación se crea en el momento en que hay una interacción de estas espirales a través del tiempo, es decir, es el proceso de la creación de conocimiento y no el conocimiento por sí solo, el que genera la innovación. Sugiriendo así, una vez más, que la innovación organizacional se crea a partir de la iteración y la interacción, esto es, procesos repetitivos y continuos, y acciones conjuntas entre diferentes actores y elementos organizacionales.

Aunado a lo anterior, y considerando que la base para la creación de conocimiento de Nonaka (1994) es el conocimiento tácito, es imperativo tener en cuenta lo mencionado por Grant (1996), quien argumenta que la estructura organizacional jerárquica no funciona cuando se trata de conocimiento tácito, puesto que este no puede ser transferido hacia arriba, lo cual hace ineficiente este tipo de coordinación. Por lo cual, una vez más, se entiende que hay una interacción entre estos elementos de la organización, y que esta debe estar dotada de cierta armonía para que la innovación se produzca.

Ahora bien, cambio e innovación son conceptos que guardan una estrecha relación, sin embargo aún no hay consenso en la manera en que se abordan los mismos, para Damanpour y Aravind (2012) existe un sistema de orden inferior que inicia con la creatividad y va avanzando con la innovación hacia un sistema de orden superior que finaliza con el cambio, de igual manera, el inicio pertenece a un ámbito individual y avanza hacia los ámbitos de equipo, unidad y, finalmente, a nivel de toda la organización con el cambio. Para Orengo, Grau, y Peiró (2002) el cambio es simplemente una nueva forma de concebir la innovación, se trata, por lo tanto, de un proceso de cambio organizacional. Mientras que para Damanpour y Evan (1984) la innovación se considera una respuesta al cambio ambiental o medios de provocar cambios en una organización.

\section{Conclusiones}

Aún cuando en la última década se ha notado un incremento acerca del interés por estudiar la innovación organizacional, falta mucho por explorar sobre este tipo de innovación, en relación con otros temas gerenciales, como la estrategia, la estructura, el aprendizaje y conocimiento organizacional, entre otros.

Las definiciones y términos utilizados para referirse a la innovación organizacional son diversos y no hay unicidad en el medio académico, por esta razón, la literatura sobre la misma se encuentra dispersa y no es posible enmarcarla dentro de un marco teórico único. 
La innovación organizacional ha sido explorada desde diferentes determinantes, tales como la estructura organizacional, el cambio y el aprendizaje organizacional, a su vez, su estudio se ha abordado tomando en cuenta diversas perspectivas.

La innovación organizacional ha sido considerada como lo soft de la innovación, en comparación con la innovación tecnológica, sin embargo, la innovación organizacional generalmente implica una relación de complementariedad para la innovación tecnológica, teniendo en cuenta que en la organización, luego de la implementación de ciertas innovaciones, es necesaria la modificación de la estructura y otros tipos de actividades que aseguren la sostenibilidad de dichas innovaciones.

\section{Referencias bibliográficas}

Argyris, C., \& Schön, D. A. (1978). Organizational learning: a theory of action perspective (Vol. 1). United States of America: Addison-Wesley Publishing Company, Inc.

Armbruster, H., Bikfalvi, A., Kinkel, S., \& Lay, G. (2008). "organizational innovation": The challenge of measuring non-technical innovation in large-scale surveys. Technovation, 28(10), 644-657.

https://doi.org/10.1016/j.technovation.2008.03.003

Birkinshaw, J., Hamel, G., \& Mol, M. J. (2008). "management innovation”. Academy of Management Review, $33(4), 825-845$.

Bovey, W. H., \& Hede, A. (2001). Resistance to organizational change: The role of cognitive and affective processes. Leadership \& Organization Development Journal, 22(8), 372-382.

https://doi.org/10.1108/01437730110410099

Buono, A. F., \& Kerber, K. W. (2010). Creating a sustainable approach to change: Building organizational change capacity. Advanced Management Journal, 4-21.

Burgelman, R. A. (1991). Intraorganizational Ecology of Strategy Making and Organizational Adaptation: Theory and Field Research. Organization Science, 2(3), 239-262. https://doi.org/10.1287/orsc.2.3.239

Burnes, B. (2005). Complexity Theories and Organizational Change. International Journal of Management Reviews, 7(2), 73-90. https://doi.org/10.1111/j.1468-2370.2005.00107.x

Calantone, R. J., Cavusgil, S. T., \& Zhao, Y. (2002). Learning orientation, firm innovation capability, and firm performance. Industrial Marketing Management, 31(6), 515-524. https://doi.org/10.1016/S00198501(01)00203-6

Camisón-Zornoza, C., Lapiedra-Alcamí, R., Segarra-Ciprés, M., \& Boronat-Navarro, M. (2004). A Meta-analysis of Innovation and Organizational Size. Organization Studies, 25(3), 331-361. https://doi.org/10.1177/0170840604040039

Camisón, C., \& Villar-lópez, A. (2012). “organizational innovation” as an enabler of technological innovation capabilities and fi rm performance is. Journal of Business Research.

https://doi.org/10.1016/j.jbusres.2012.06.004

Damanpour, F., \& Aravind, D. (2011). "managerial innovation": Conceptions, Processes, and Antecedents. Management and Organization Review, 8(2), 423-454. https://doi.org/10.1111/j.1740-8784.2011.00233.x 
Damanpour, F., \& Aravind, D. (2012). Organizational Structure and Innovation Revisited : From organic to ambidextrous structure. Handbook of Organizational Creativity. Elsevier Inc.

https://doi.org/10.1016/B978-0-12-374714-3.00019-7

Damanpour, F., \& Evan, W. M. (1984). "organizational innovation" and Performance: The problem of "Organizational Lag ". Administrative Science Quarterly, 29(3), 392-409.

Donaldson, L. (2001). The Contingency theory of Organizations. London: Sage Publications Ltd.

Donaldson, L. (2008). The Conflict Between Contingency and Institutional Theories of Organizational Design. In R. M. Burton, D. D. Håkonsson, C. C. Snow, B. H. Eriksen, \& T. Knudsen (Eds.), Designing Organizations (p. 240). New York: Springer.

Donaldson, L. (2015). Structural Contingency Theory. International Encyclopedia of the Social \& Behavioral Sciences: Second Edition, 23, 609-614. https://doi.org/10.1016/B978-0-08-097086-8.73110-2

Drucker, P. F. (2005). La nueva sociedad de las organizaciones. Harvard Business Review América Latina, 9.

Evan, W. (1966). Organizational Lag. Human Organization, 25(1), 51-53. https://doi.org/10.17730/humo.25.1.v7354t3822136580

Galbraith, J. R. (2014). Designing organizations: Strategy, structure, and process at the business unit and enterprise levels (3rd ed.). San Francisco: Jossey- Bass.

García-Cabrera, A. M., Álamo-Vera, F. R., \& Hernández, F. G. B. (2011). Antecedentes de la resistencia al cambio: Factores individuales y contextuales. Cuadernos de Economia y Direccion de La Empresa, 14(4), 231-246. https://doi.org/10.1016/j.cede.2011.02.007

Garcilazo, J. (2011). LA TEORÍA DE LA ECOLOGÍA POBLACIONAL EXAMINADA A LA LUZ DE TEORÍAS ADAPTACIONISTAS. Visión de Futuro, 15(1).

Glynn, M. A. (1996). Innovative Genius : A Framework for Relating Individual and Organizational Intelligences to Innovation. Academy of Management, 21(4), 1081-1111.

Grant, R. M. (1996). Toward a knowledge-based theory of the firm. Strategic Management Journal, 17(Winter Special Issue).

Gu, W., \& Surendra, G. (2004). The Effect of "organizational innovation" and Information Technology on Firm Performance. Ssrn. https://doi.org/10.2139/ssrn.1404689

Hannan, M. T., \& Freeman, J. (1977). The Population Ecology of Organizations. American Journal of Sociology, 82(5), 929-964.

Hendrickson, S., \& Gray, E. J. (2012). Legitimizing Resistance to Organizational Change : A Social Work Social Justice Perspective. International Journal of Humanities and Social Science, 2(5), 50-59.

Kerber, K., \& Buono, A. (2005). RETHINKING ORGANIZATIONAL CHANGE : Reframing the Challenge of Change Management. Organizational Development Journal, 23(3), 23-38.

Lam, A. (2004). "organizational innovation". In J. FAGERBERG, D. C. MOWERY, \& R. R. NELSON (Eds.), The Oxford Handbook of Innovation (p. 674). Oxford University Press. https://doi.org/10.1016/S13872656(08)70001-2 
Lawrence, P. R., \& Lorsch, J. W. (1967). Differentiation and Integration in Complex Organizations. Administrative Science Quarterly, 12(1), 1. https://doi.org/10.2307/2391211

Li, M., \& Gao, F. (2003). Why Nonaka highlights tacit knowledge: A critical review. Journal of Knowledge Management, 7(4), 6-14. https://doi.org/10.1108/13673270310492903

Liao, S. H., \& Wu, C. chuan. (2010). System perspective of knowledge management, organizational learning, and "organizational innovation". Expert Systems with Applications, 37(2), 1096-1103. https://doi.org/10.1016/j.eswa.2009.06.109

Lines, R. (2005). The Structure and Function of Attitudes Toward Organizational Change. Human Resource Development Review, 4(1), 8-32. https://doi.org/10.1177/1534484304273818

March, J. G. (1981). Footnotes to Organizational Change. Administrative Science Quarterly, 26(4), 563-577.

Marín-Idárraga, D. A., \& Cuartas, J. C. (2016). Organizational structure and innovation: analysis from the strategic co-alignment. Academia Revista Latinoamericana de Administración, 29(4), 388-406. https://doi.org/10.1108/arla-11-2015-0303

Menguc, B., \& Auh, S. (2010). Development and return on execution of product innovation capabilities: The role of organizational structure. Industrial Marketing Management, 39(5), 820-831. https://doi.org/10.1016/j.indmarman.2009.08.004

Merx-Chermin, M., \& Nijhof, W. J. (2005). Factors influencing knowledge creation and innovation in an organisation. Journal of European Industrial Training, 29(2), 135-147. https://doi.org/10.1108/03090590510585091

Mintzberg, H. (1981). Organization Design: Fashion or Fit? Harvard Business Review.

Nonaka, I. (1994). A Dynamic Theory of Organizational Knowledge Creation. Organization Science, 5(1), 14-37. https://doi.org/10.1287/orsc.5.1.14

Nonaka, I., \& Takeuchi, H. (1995). La Organización Creadora De Conocimiento: Cómo las compañías japonesas crean la dinámica de la innovación. La organización creadora del Conocimiento. México D.F: Oxford University Press.

OECD. (2005). Oslo manual. Retrieved from http://www.oecd.org/science/inno/2367580.pdf

Ogbonna, E., \& Harris, L. C. (2003). Innovative organizational structures and performance: A case study of structural transformation to "groovy community centers. Journal of Organizational Change Management, 16(5), 512-533. https://doi.org/10.1108/09534810310494919

Olson, E. M., Slater, S. F., \& Hult, G. T. M. (2005). The Performance Implications of Fit Among Business Strategy, Marketing Organization Structure, and Strategic Behavior. Journal of Marketing, 69(3), 49-65. https://doi.org/10.1509/jmkg.69.3.49.66362

Orengo, V., Grau, R., \& Peiró, J. M. (2002). La innovación tecnológica como proceso de cambio organizacional. Revista de Psicología Del Trabajo y de Las Organizaciones, 18(1), 5-38.

Pawlowski, E. (2016). Flexibility of Organizational Structure in a Context of "organizational innovation"s and Modern Concepts of Enterprise Management. Technology Management for Social Innovation, 2331-2337.

Pfeffer, J. (1997). Nuevos rumbos en la teoría de la organización. Problemas y posibilidades. México D.F: Oxford University Press. 
Poole, M. S. (2004). Central Issues in the Study of Change and Innovation. In M. S. Poole \& A. H. Van de Ven (Eds.), Handbook of organizational change and innovation (Vol. 42, pp. 42-5357-42-5357). New York: Oxford University Press. https://doi.org/10.5860/choice.42-5357

Rezaei, A., Allameh, S. M., \& Ansari, R. (2018). Impact of knowledge creation and organisational learning on organisational innovation: an empirical investigation. International Journal of Business Innovation and Research, 16(1), 117. https://doi.org/10.1504/ijbir.2018.091087

Romero, J., Matamoros, S., \& Campo, C. A. (2013). Sobre el cambio organizacional. Una revisión bibliográfica. Innovar Journal, 23(50), 35-52.

Sandoval Duque, J. L. (2014). Los procesos de cambio organizacional y la generación de valor. Estudios Gerenciales, 30(131), 162-171. https://doi.org/10.1016/j.estger.2014.04.005

Schoonhoven, C. B. (1981). Problems with contingency theory: Testing assumptions hidden within the language of contingency "theory." Administrative Science Quarterly, 26, 349-377. https://doi.org/10.2307/256633

Senge, P. M. (1990). The fifth discipline: The art and practice of the learning organization. New York: Doubleday.

Simao, L., \& Franco, M. (2018). External knowledge sources as antecedents of "organizational innovation" in fi rm workplaces : a knowledge-based perspective. Journal of Knowledge Management, 22(2), 237-256. https://doi.org/10.1108/JKM-01-2017-0002

Su, Z., Chen, J., \& Wang, D. (2018). Organisational structure and "managerial innovation": the mediating effect of cross-functional integration. Technology Analysis and Strategic Management, 31(3), 253-265. https://doi.org/10.1080/09537325.2018.1495324

Sutanto, E. M. (2017). The influence of organizational learning capability and organizational creativity on "organizational innovation" of Universities in East Java, Indonesia. Asia Pacific Management Review, 22(3), 128-135. https://doi.org/10.1016/j.apmrv.2016.11.002

Tohirovich, A., Rhee, C., \& Yoon, J. (2017). Organizational structure and innovation performance Is employee innovative behavior a missing link? Career Development International, 22(4), 334-350. https://doi.org/10.1108/CDI-12-2016-0234

West, M. A., \& Farr, J. L. (1989). Innovation at work: Psychological perspectives. In D. K. Simonton, M. A. West, \& J. L. Farr (Eds.), Innovation and Creativity at Work: Psychological and Organizational Strategies. (pp. 313). Willey.

Wolfe, R. A. (1994). "organizational innovation": Review, Critique and Suggested Research Directions. Journal of Management Studies, 31(3), 405-461. Retrieved from https://onlinelibrary-wileycom.manchester.idm.oclc.org/doi/pdf/10.1111/j.1467-6486.1994.tb00624.x

Woodman, R. W., Sawyer, J. E., \& Griffin, R. W. (1993). Towards a Theory of Organizational Creativity. Academy of Management Review, 18(2), 293-321. https://doi.org/10.2307/258761 